\title{
Transcranial Ultrasound Stimulation of the Anterior Cingulate Cortex Reduces Neuropathic Pain in Mice
}

\author{
Xiangjun Feng, ${ }^{1,2,3}$ Lili Niu, ${ }^{3,4,5}$ Meng Long, ${ }^{3,4,5}$ Kaixuan Luo $\mathbb{D}^{1,3}$ Xiaowei Huang, \\ Moxian Chen, ${ }^{1}$ Zhengrong Lin, ${ }^{3}$ Wei Zhou, ${ }^{3}$ Shasha Yi, ${ }^{1,3}$ and Lijuan Ao ${ }^{1}{ }^{1}$ \\ ${ }^{1}$ The School of Rehabilitation, Kunming Medical University, 1168 West Chunrong Road, Chenggong, Kunming 650500, China \\ ${ }^{2}$ General Surgery Department of Geriatrics, The First Affiliated Hospital of Kunming Medical University, 295 Xichang Road, \\ Kunming 650032, China \\ ${ }^{3}$ Institute of Biomedical and Health Engineering, Shenzhen Institutes of Advanced Technology, Chinese Academy of Sciences, \\ 1068 Xueyuan Avenue, Shenzhen 518055, China \\ ${ }^{4}$ CAS Key Laboratory of Health Informatics, Shenzhen Institutes of Advanced Technology, 1068 Xueyuan Avenue, \\ Shenzhen 518055, China \\ ${ }^{5}$ Guangdong-Hong Kong-Macao Greater Bay Area Center for Brain Science and Brain-Inspired Intelligence, \\ 1068 Xueyuan Avenue, Guangzhou 518055, China
}

Correspondence should be addressed to Lijuan Ao; aolijuan69@gmail.com

Received 10 August 2021; Accepted 22 November 2021; Published 31 December 2021

Academic Editor: Feng Zhang

Copyright $\odot 2021$ Xiangjun Feng et al. This is an open access article distributed under the Creative Commons Attribution License, which permits unrestricted use, distribution, and reproduction in any medium, provided the original work is properly cited.

Focused ultrasound (FUS) is a potential tool for treating chronic pain by modulating the central nervous system. Herein, we aimed to determine whether transcranial FUS stimulation of the anterior cingulate cortex (ACC) effectively improved chronic pain in the chronic compress injury mice model at different stages of neuropathic pain. The mechanical threshold of pain was recorded in the nociceptive tests. We found FUS stimulation elevated the mechanical threshold of pain in both short-term $(p<0.01)$ and longterm $(p<0.05)$ experiments. Furthermore, we determined protein expression differences in ACC between the control group, the intervention group, and the Sham group to analyze the underlying mechanism of FUS stimulation in improving neuropathic pain. Additionally, the results showed FUS stimulation led to alterations in differential proteins in long-term experiments, including cellular processes, cellular signaling, and information storage and processing. Our findings indicate FUS may effectively alleviate mechanical neuropathic pain via the ACC's stimulation, especially in the chronic state.

\section{Introduction}

Chronic pain is a severe condition that considerably interferes with daily functioning [1], and the estimated prevalence of chronic pain ranges from $8.7 \%$ to $64.4 \%[2,3]$. Neuropathic pain (NP) is a prominent type of chronic pain. Chronic pain causes not only stress on the body, including strained muscles, diminished motion range, inadequate power, and appetite changes, but also emotional effects, such as depression, anger, anxiety, and fear of reinjury, which may limit the ability to return to routine work or leisure activities [4]. The treatment is mainly medication or physical therapy, but the effect is not significant due to the complicated etiology
$[5,6]$. Therefore, it is necessary to develop effective strategies to improve these issues.

The use of the S-size ultrasound probe in pulsed mode stimulation over a skin incision has been reported to improve the mechanical and thermal retraction threshold of the NP model [7]. A previous study reported that axonal regeneration in autograft nerves was improved following low-intensity pulsed ultrasound with $250 \mathrm{~mW} / \mathrm{cm}^{2}$ compared with that following high-intensity ultrasound [8]. Therefore, focused ultrasound (FUS) may be a nonpharmacological nonablative neuromodulatory technique that improves peripheral nerve injury or NP. Additionally, Hameroff et al. stimulated individuals with chronic pain using transcranial ultrasound and 
reported an unexpected analgesic effect [9]. Moreover, Spooner et al. reported that deep brain stimulation over the bilateral cingulate by $130 \mathrm{~Hz}$ high-frequency electricity resulted in pain relief in a patient with severe drug-resistant pain syndrome following a complete spinal cord injury [10]. Subsequently, there has been increasing interest in modulating the central nervous system (CNS) for chronic pain treatment.

Thalamus is an important regulatory target for the treatment of pain, and ACC is a possible target for pain management through previous researches. Pain relief using cingulotomy has evoked clinical interest in deep brain stimulation in the dorsal ACC for treating chronic refractory pain, especially when coupled with a substantial affective component, such as distress, resulting in the more complicated treatment [11]. ACC activation improves chronic pain states through several neuronal modulation changes in the CNS [12-14]. Moon et al. reported that optical inhibition of the ACC improved pain-associated behavior and reduced the unusual activity of thalamic sensory neurons in a rat model of trigeminal NP [15]. In addition, various neuromodulation techniques have demonstrated therapeutic value against NP by inhibiting neuronal activity in the ACC, a crucial target in the brain $[13,15-17]$.

At present, deep brain stimulation, transcranial magnetic stimulation, and transcranial electrical stimulation are the most commonly used regulation programs of the CNS, which may have the disadvantages of high surgical risk and poor accuracy. Recent studies have also shown that FUS is a safe, noninvasive, and accurate technique that modulates neuronal circuits in the CNS $[18,19]$ of both animal models and humans [20-22]. Moreover, transcranial FUS may treat chronic pain through neuronal regulation of the CNS, including the ACC. However, the treatment effect of FUSinduced ACC activation on chronic pain remains unclear. Consequently, we investigated the therapeutic effects of FUS stimulation of the ACC using the short- and long-term NP chronic compress injury (CCI) mouse model.

\section{Materials and Methods}

2.1. Animal Preparation. We conducted all animal experiments based on the guidelines approved by the Animal Use Committee and the Ethics Committee of Kunming Medical University (Approval number: KMMU2019075). For shortterm experiments, we randomly allocated $18 \mathrm{C} 57 \mathrm{BL} / 6 \mathrm{~J}$ mice (age: 8 weeks, weight: $20-23 \mathrm{~g}$, male) to the FUS1 (parameter 1), FUS2 (parameter 2), and control (sham stimulation) groups ( $n=6$ mice/group). For the long-term experiments, we randomly allocated $36 \mathrm{C} 57 \mathrm{BL} / 6 \mathrm{~J}$ mice (age: 14 months, weight: 24-36 g, female) to the Control (Sham operation), FUS (parameter 2), and Sham (sham stimulation) groups ( $n=12$ mice/ group). All animals were raised in a controlled environment $\left(22 \pm 2^{\circ} \mathrm{C}\right)$ under a regular light-dark cycle (lights on, 7 a.m.; lights off, 7 p.m.) with ad libitum access to food and water.

2.2. Chronic Pain Model. Following a 1-week acclimation of the mice to the maintenance environment, we began the experiment. We used the CCI surgical procedure to establish the NP model [23]. We conducted nociceptive tests, including the mechanical allodynia test, thermal allodynia test, and sciatic nerve functional index, to obtain baseline values before the CCI surgical procedure on the right sciatic nerve. The mice were anesthetized with isoflurane $(2 \%$ for induction and $1.5 \%$ for maintenance, Sigma-Aldrich, St. Louis, Missouri, USA) and placed in the prone position. Next, the right thigh was shaved, and the skin was disinfected with $2 \%$ iodinealcohol. After a skin incision was made in the middle third of the right hind limb to expose the biceps femoris muscle, approximately $5 \mathrm{~mm}$ of the sciatic nerve was uncovered, and three ligatures (gut ligatures 6.0, Jinhuang, Shanghai, China) were tied at $1-\mathrm{mm}$ intervals. The tying allowed noticeable nerve constriction without arresting epineurial blood flow. The skin was sutured using Mononylon 4.0 (Johnson \& Johnson Medical N.V., Belgium, 2018-2019). Mice in the sham-operated group were operated according to the abovementioned surgical procedure, but after the nerve was exposed, an intestinal ligature was placed on the sciatic nerve trunk three times without ligation; the nerve was repositioned, and the skin was sutured We returned the animals to their cages after surgery and recovery from anesthesia.

2.3. Nociceptive Tests. We conducted nociceptive tests before (baseline) and following surgery in all experimental groups. In the short-term experiment, we started ultrasound stimulation when there was a substantial decrease in the mechanical pain threshold of the entire group $(\approx 0.008 \mathrm{~g} ; \approx 6$ days following surgery). A substantial reduction in the mechanical pain threshold during the postoperative examination indicated successful establishment of the pain model in the long-term experiment. We started ultrasound stimulation on the $91^{\text {st }}$ days after the surgery and repeated the nociceptive tests every 6-7 days following ultrasound stimulation commencement.

2.3.1. Mechanical Allodynia Test. All the animals were allowed 2-3 days to acclimate to the test environment before the tests or surgery. We placed the mice in a ten-grid Plexiglas box (homemade) with a metal net lid and bottom for about $30 \mathrm{~min}$. Next, we perpendicularly stimulated the bilateral hind paws on the plantar surface in an ascending order of stimulus intensity using Von Frey hairs (Aesthesio, DanMic Global, Campbell, CA) with increasing stiffness $(0.008,0.02,0.04,0.07,0.16,0.4,0.6,1,1.4,2$, and $4 \mathrm{~g})$. A positive situation was defined when the mice ran away or raised the hind leg following a 5-6 s perpendicular stimulation. We recorded the gradually growing stiffness when a positive result occurred three times in five stimulations separated by more than $3 \mathrm{~min}$. We repeated the process mentioned above 3-5 times at intervals of $>15 \mathrm{~min}$ and obtained the mean value as the mechanical withdrawal threshold to indicate the mechanical pain tolerance of mice.

2.3.2. Thermal Allodynia Test. We used a laser transmitter (ADR-1805, Xilongguangdian, Shanghai, China) to determine the thermal allodynia value with a near-infrared laser 
(wavelength, $787.7 \mathrm{~nm}$; output power, $564 \mathrm{~mW}$ ) that was calibrated using an infrared thermal imager (R300, NEC Avio, Tokyo, Japan). The calibration warmed the paw sole skin to $50^{\circ} \mathrm{C}$ at a distance of $0.5 \mathrm{~cm}$ within $28 \mathrm{~s}$. The mice were allowed to habituate to the Plexiglas box for at least $30 \mathrm{~min}$. The light was transmitted to the paw sole skin using the laser transmitter at a distance of approximately $0.5 \mathrm{~cm}$. The time from the start of lighting to paw withdrawal was recorded with a 60-s limit to avoid local burn injury. This described procedure was performed twice at a 15 -min interval, and the mean value was used as the thermal withdrawal threshold to indicate the thermal pain tolerance of mice.

2.3.3. Sciatic Nerve Functional Index (SFI). We prepared a plastic corridor $(5 \mathrm{~cm} \times 10 \mathrm{~cm} \times 15 \mathrm{~cm})$ with paper tape $(6 \mathrm{~cm} \times 20 \mathrm{~cm}$, homemade) at the bottom and an ink pad at the beginning of the corridor for the mice to walk through. The mice were briefly placed on the ink pad and walked straight through the corridor while leaving footprints on the paper tape prior to and following the CCI surgery. When there were three clear footprints, we measured the following three parameters: print length (PL), distance from the heel to the third toe; toe spread (TS), distance from the first toe to the fifth toe; and intermediate TS (ITS), distance from the second toe to the fourth toe. We calculated the SFI using the following formula [24]:

$$
\begin{aligned}
\mathrm{SFI}= & 38.3\left(\frac{\mathrm{EPL}-\mathrm{NPL}}{\mathrm{NPL}}\right)+109.5\left(\frac{\mathrm{ETS}-\mathrm{NTS}}{\mathrm{NTS}}\right) \\
& +13.3\left(\frac{\text { EITS }-\mathrm{NITS}}{\mathrm{NITS}}\right)-8.8
\end{aligned}
$$

where $E$ represents the experimental side and $N$ represents the normal side. A value of 0 indicates the normal function, and -100 indicates total impairment.

2.4. FUS Stimulation Process. We used an arbitrary waveform generator (3102C, Tektronix, Texas, USA) with a power amplifier (LZY-22+, Mini-circuits, Shenzhen, China) to provide an output to apply to a $3.7 \mathrm{MHz}$ focused transducer with a focal diameter and length of 0.7 and $11.1 \mathrm{~mm}$, respectively. We performed FUS stimulation of the ACC $(\mathrm{Cg})$ region of mice to induce pain relief in the mice (the ultrasound pulse parameters were as follows: pulse repetition frequency, $1.5 \mathrm{kHz}$ and duty cycle, $10 \%$. The spatial-peak pulse-averaged acoustic intensity (Isppa) was $15980 \mathrm{~mW} /$ $\mathrm{cm}^{2}$ and $34982 \mathrm{~mW} / \mathrm{cm}^{2}$ for FUS1 and FUS2, respectively. Moreover, the spatial-peak temporal-average intensity (Ispta) was $1598 \mathrm{~mW} / \mathrm{cm}^{2}$ and $3498 \mathrm{~mW} / \mathrm{cm}^{2}$ for FUS1 and FUS2, respectively (Figure 1(a)). These values were obtained using degassed water through an ultrasound test tank system (Precision Acoustics Ltd., Dorchester, United Kingdom) equipped with a calibrated hydrophone (2010, Precision Acoustics Ltd., Dorchester, United Kingdom). We measured the acoustic intensity attenuation through the skull up to $80 \%$, and the focal spot can be located just below the skull.
The measured acoustic field distribution shows the measured acoustic pressure of the probe, with or without a fresh mouse skull. The focus distance for projecting across the cingulate cortex area through the collimator filled with degassed water was $11.1 \mathrm{~mm}$ (Figure 1(b)).

All the mice were depilated using a depilatory paste (Veet, Reckitt Benckiser, Hubei, China), and we used acoustic coupling gel for FUS treatment $(15 \mathrm{~min} /$ day for 21 days). The mice underwent ultrasonic stimulation under anesthesia with isoflurane (2\% for induction, $0.6 \%$ for maintenance, Sigma-Aldrich, St. Louis, Missouri, USA). The mice were depilated and anesthetized; then, the head of the mice was fixed on the mouse-adapted stereotaxic device. The collimator prepared by calculating the focal length of the ultrasound transducer was placed directly above the brain area of ACC (bregma point) and was coupled with a coupling agent to ensure no air was left (Figure 1(a)). Ultrasound stimulation was performed through the collimator, just touching the scalp of the mice using the above parameters, and the sham procedure was performed at the same time, which involved the same procedure of all working processes for equal time, with all equipment turned on, without ultrasonic excitation signal.

First, we performed FUS on the short-term pain model to determine the effect of FUS on the ACC brain area in the early stage of pain. On the 6th day after surgery, we confirmed the successful establishment of the CCI model using the nociceptive tests. On the 7th day, we started FUS stimulation at $15 \mathrm{~min} /$ day for 21 days (Figure 2(a)). After completing the short-term experiment, we confirmed that FUS has a better neuromodulation effect when using the FUS2 parameters. Therefore, we conducted long-term experiments to confirm the effectiveness of the ACC regulation with FUS in the chronic pain period using FUS2 parameters. Following successful establishment of the CCI model, the mice were allowed to move freely for 90 days without intervention, and the baseline values of nociceptive tests were determined prior to ultrasound stimulation. On the 91st day, we started the FUS2 stimulation using a similar protocol to that used for the shortterm experiment (Figure 3(a)).

2.5. Slice Preparation and Multielectrode Array (MEA) Recordings. To verify the direct effect of ultrasound on neurons in the ACC brain area using FUS2 parameters, we separately recorded three brain slices of three C57BL/6J mice for MEA recordings. The animals were sacrificed using a rodent guillotine (RWD, Shenzhen, China) under deep anesthesia with $5 \%$ isoflurane. Next, we collected the brains and placed them in an ice-cold oxygenated high-sucrose cutting solution $\left(0-2^{\circ} \mathrm{C}\right)$ that contained the following ingredients (in mM): $60 \mathrm{NaCl}, 3 \mathrm{KCl}, 7 \mathrm{MgCl}_{2}, 1.25 \mathrm{NaH}_{2} \mathrm{PO}_{4}$, $25 \mathrm{NaHCO}_{3}, 10 \mathrm{D}$-glucose, 115 sucrose, and $0.5 \mathrm{CaCl}_{2}$. Subsequently, we prepared $500 \mu \mathrm{m}$ coronal slices of the ACC area using a vibratome (VT-1200 Series, Leica Biosystems, Wetzlar, Germany). The slices were then equilibrated and incubated in artificial cerebrospinal fluid (ACSF) that contained the following ingredients (in $\mathrm{mM}$ ): $126 \mathrm{NaCl}, 2.5$ 

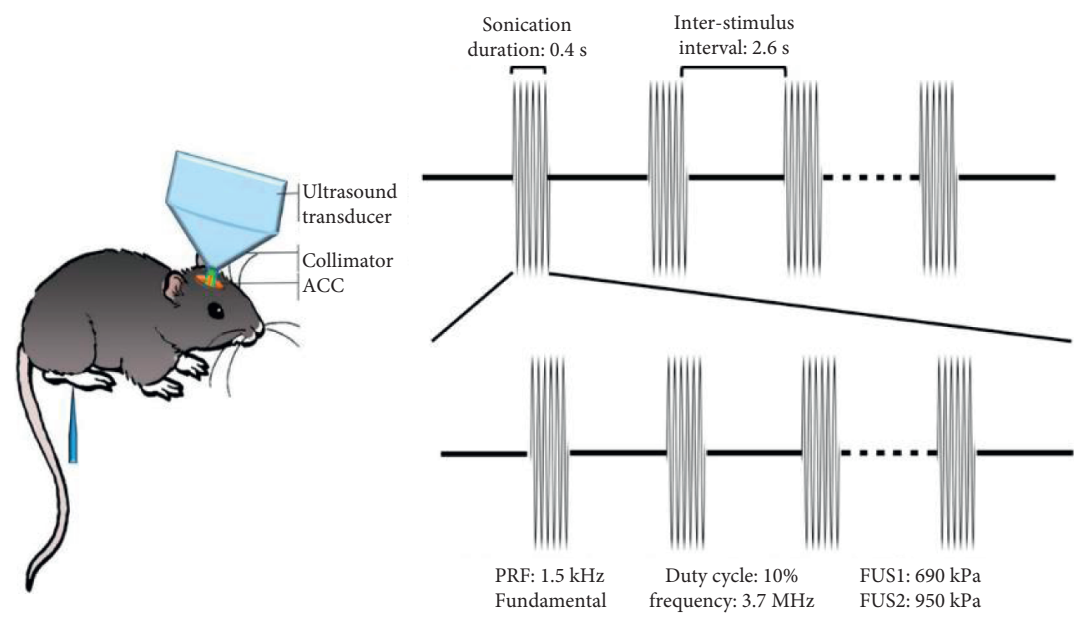

(a)
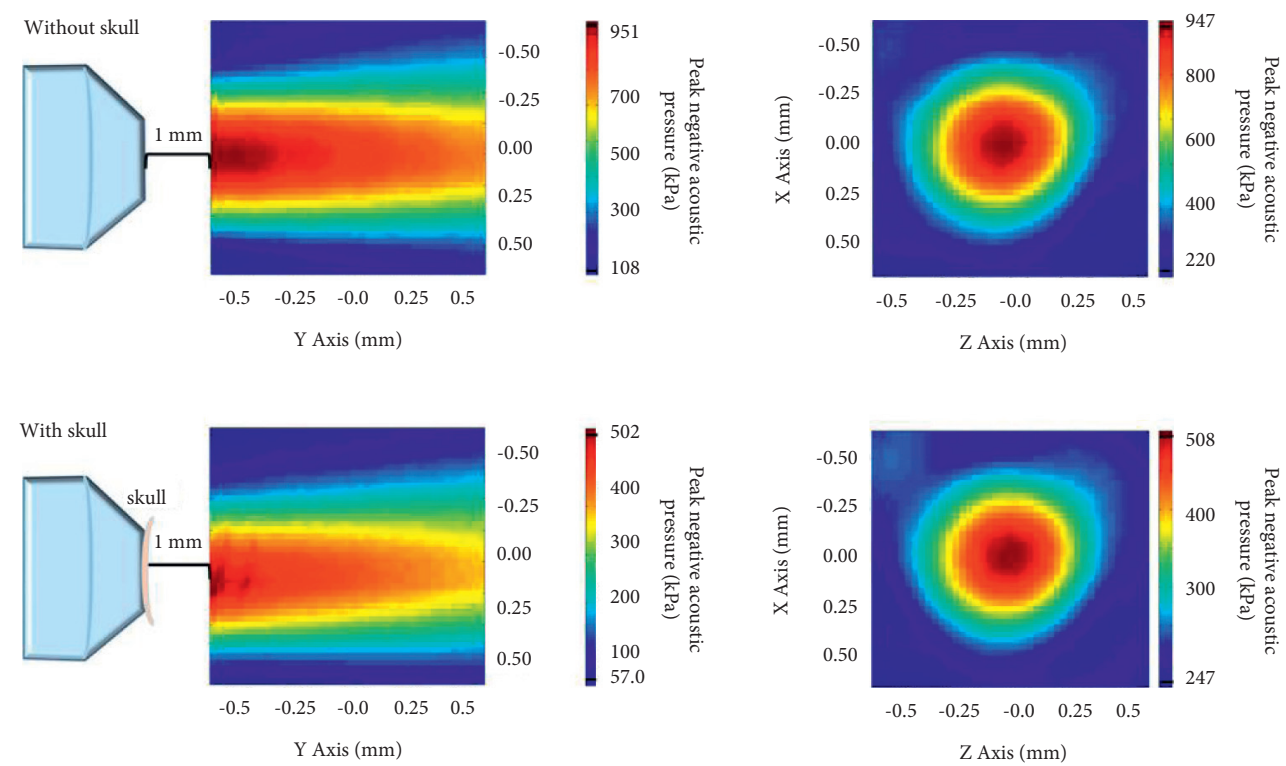

(b)

Figure 1: Experimental schematics. Experimental schematic diagram (a). Acoustic field distributions with or without a fresh mouse skull (b).

$\mathrm{KCl}, 1 \mathrm{MgCl}_{2}, 1.25 \mathrm{NaH}_{2} \mathrm{PO}_{4}, 26 \mathrm{NaHCO}_{3}, 10 \mathrm{D}$-glucose, 2 sodium pyruvate, $0.5 \mathrm{~L}$-ascorbic acid, and $2 \mathrm{CaCl}_{2}$. The ACSF was continuously saturated with $95 \% \mathrm{O}_{2}-5 \% \mathrm{CO}_{2}$ and maintained at a temperature of $35^{\circ} \mathrm{C}$ (all the above regents: Sigma-Aldrich, St. Louis, Missouri, USA).

To investigate the FUS-induced modulatory effects, we recorded spikes from the slices using the MEA systems (MCS MEA 2100-IFB, MCS, Reutlingen, Germany), which reliably quantifies neuronal activity $[25,26]$. The brain slices were placed in the recording chamber and continuously perfused with ACSF saturated with 95\% $\mathrm{O}_{2}-5 \% \quad \mathrm{CO}_{2}$ (Figure 4(a)). We applied a solution with higher potassium levels $(2.5 \mathrm{mM}$ to $5 \mathrm{mM}$ of $\mathrm{KCl}$ in the ACSF) to evoke more discharge from the brain slices. We placed an ultrasound transducer with a coupling cone (homemade) over the ACC slices and adjusted its location to ensure the acoustic field was focused on the stimulation site of the slices. We recorded the baseline value at $100 \mathrm{~s}$ before the ultrasound intervention. Following the record of 60-s intervention (parameter 2 ), we subsequently recorded the measurement value of another $100 \mathrm{~s}$ without intervention and identified effective neuronal discharge as those with amplitude 2.5 times greater than the baseline amplitude. There were a total of 29 channels of electrical signals recorded through MEA, indicating that the excitability of 29 neuron cells was recorded. The effective discharge times were counted manually for statistical analysis.

2.6. Detection of Proteins. To preliminarily determine the local protein expression changes of ACC after the neuroregulation of FUS and to understand the possible central control mechanism of pain, we conducted protein detection on the ACC brain area of the long-term experimental group 


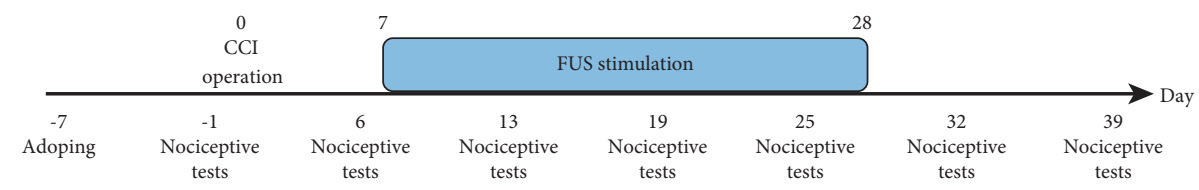

(a)
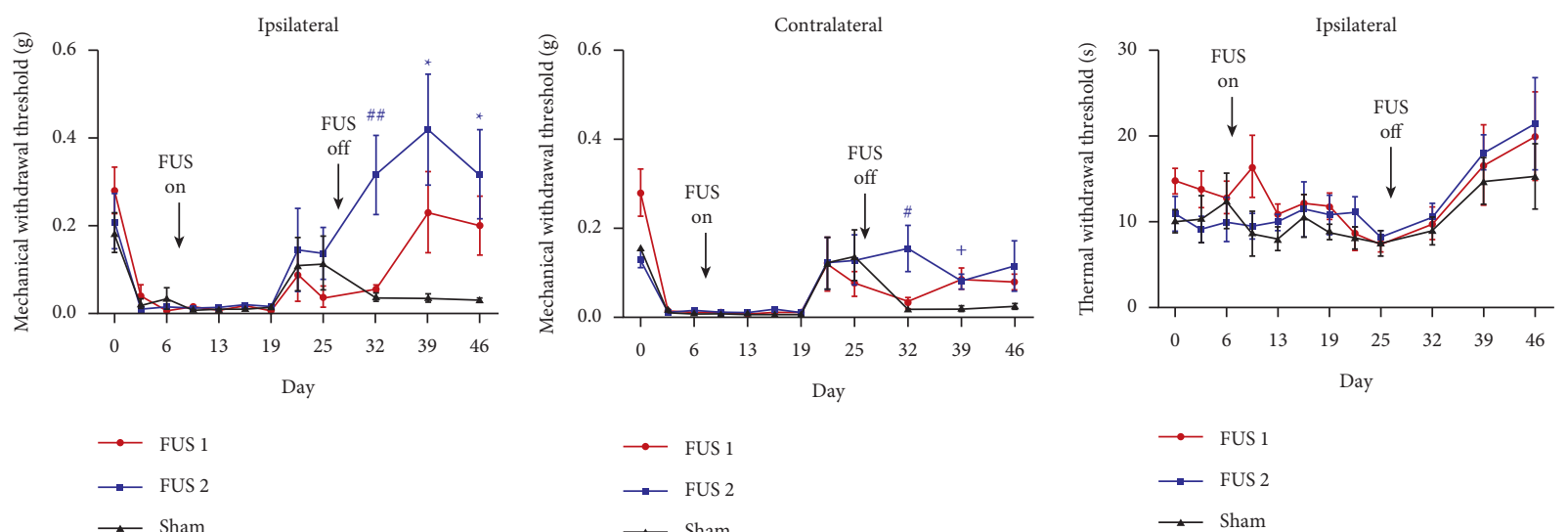

(b)

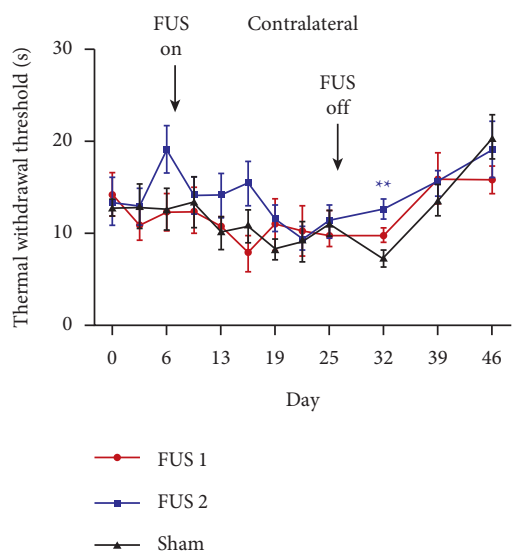

(c)

(d)
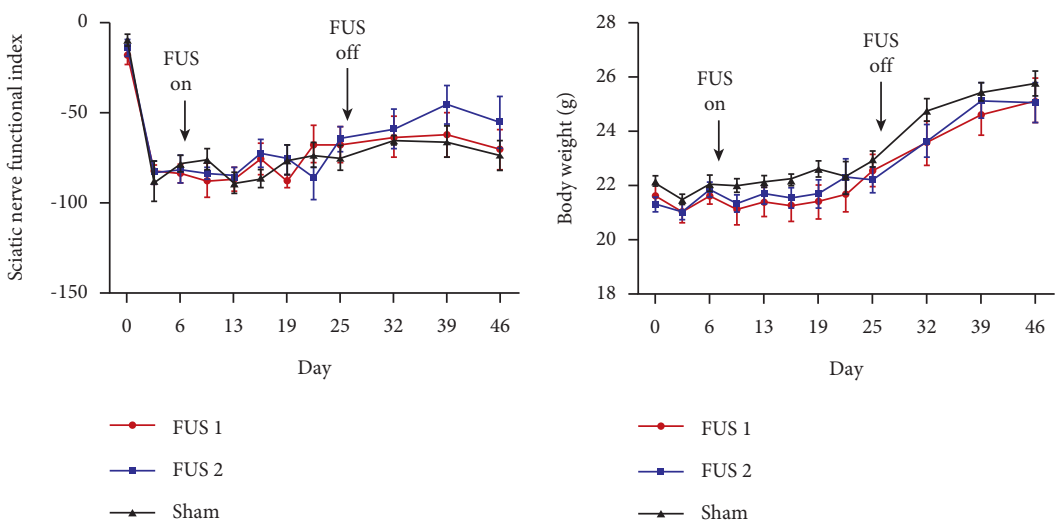

(e)

(f)

(g)

FIgURE 2: Transcranial FUS stimulation slowly improved the mechanical withdraw threshold in the short-term experiment. Timeline of the short-term experiment $(n=6)$ (a). Ultrasound stimulation increased the mechanical withdrawal thresholds of the surgical side $\left({ }^{\# \#} p=0.003\right.$ ) (b). The increase in the mechanical and thermal withdrawal thresholds of the contralateral side (c, e). The variation of sciatic nerve index and body weight $(\mathrm{f}, \mathrm{g}){ }^{*}$ represents a statistically significant difference between the FUS2 group and the other two groups. * indicates a statistically significant difference between the FUS2 group and the Sham group. + indicates a statistically significant difference between the FUS1 group and the Sham group.

using an Isobaric tag for relative and absolute quantitation (iTRAQ), which is among the most commonly used methods in quantitative proteomics research. It works on the principle of the reaction of digested polypeptides from different samples with differentially labeled iTRAQ reagents [27]. On the 21 st day of the long-term experiment, 12 mice ( $n=6$ /group) were cardiacally perfused with a saline solution. Subsequently, the whole brains were quickly removed, and the entire ACC tissue was obtained according to the map [28]. Following multiple sample protein extractions, we performed protein digestion and quantification based on the filter-aided sample preparation method [29]. Further, we vacuum-dried (Savant DNA120, Thermo Scientific, Massachusetts, USA) three samples with the greatest protein concentration in each group for iTRAQ-based identification. We used information-dependent acquisition (IDA) mass spectrum techniques to obtain tandem mass spectrometry (MS) data on a ThermoFisher $Q$ Exactive mass spectrometer (Thermo Scientific, Massachusetts, USA) fitted with a Nano Flex ion source (Thermo Scientific, Massachusetts, USA). We performed the FULL-MS scans with an ion spray voltage of $1.9 \mathrm{kV}$ and an interface heater temperature of $275^{\circ} \mathrm{C}$. We obtained survey scans of IDA within $250 \mathrm{~ms}$ and a maximum of 20 production scans within $50 \mathrm{~ms}$. With a dynamic exclusion of $25 \mathrm{~s}$, fragmentation was performed with higherenergy collision energy dissociation for $2+$ to $4+$ charged spectra. We analyzed the MS/MS data for protein identification and quantification using IPeak. 


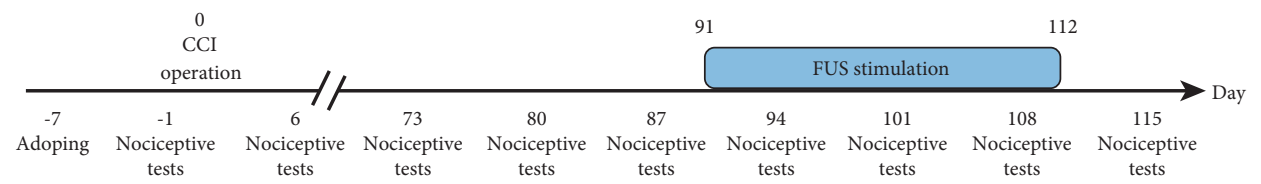

(a)

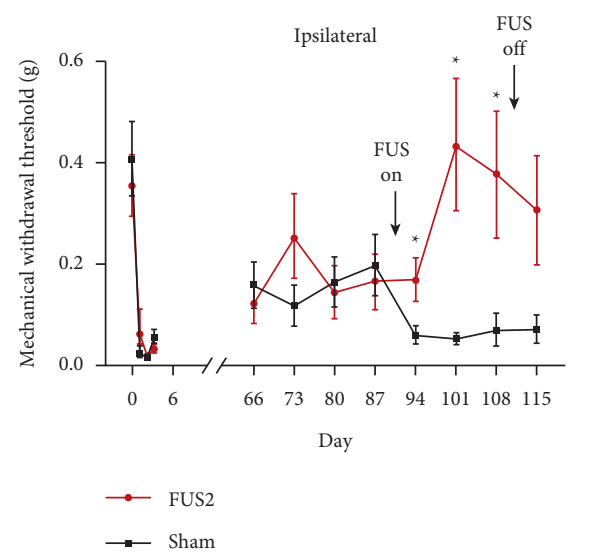

(b)

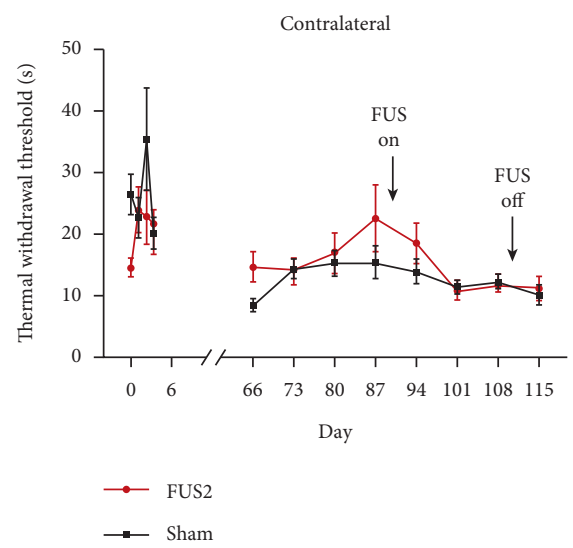

(e)

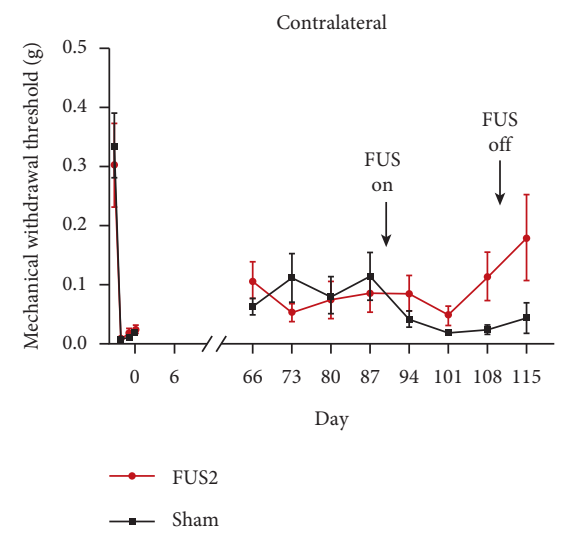

(c)

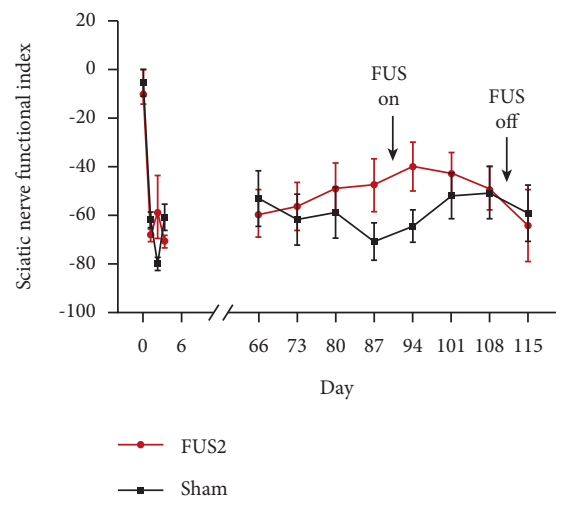

(f)

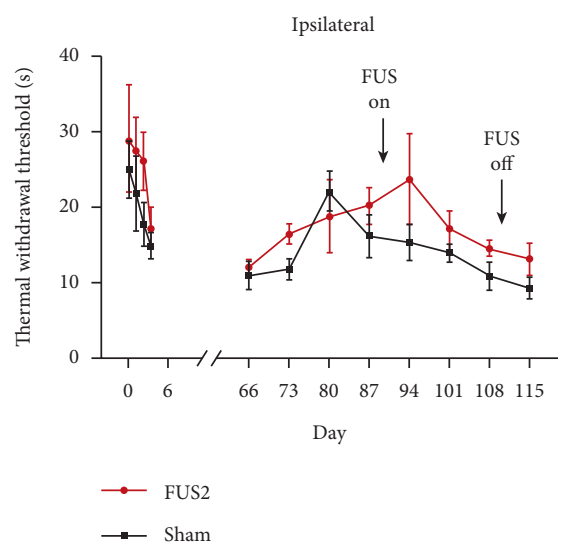

(d)

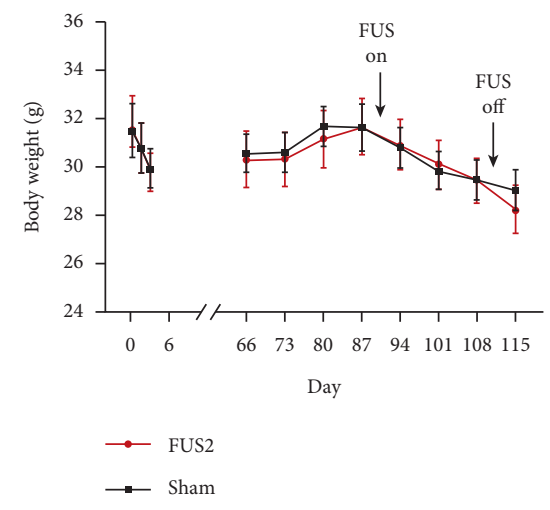

(g)

FIGURE 3: FUS stimulation promptly improved the mechanical withdraw threshold in the long-term experiment. Timeline of the long-term experiment $(n=12)$ (a). Ultrasound stimulation increased the mechanical withdraw threshold of the surgical side $(p<0.05)(\mathrm{b})$. There were no significant changes in the mechanical withdraw threshold of the contralateral side. The bilateral thermal withdraw threshold, body weight, and sciatic nerve index between the FUS2 group and the Sham group (c-f). ${ }^{*}$ indicates statistical significance between the FUS2 group and the Sham group.

2.7. Western Blotting. Total protein was extracted from tissues. Antibodies Hnrnph1, Snrpb, Dhx16, and GAPDH were purchased from Abcam (USA). Antibody Hnrnpd was purchased from CST (USA). Membranes were blocked with $5 \%$ milk and incubated with the primary antibodies in 5\% bovine serum albumin (BSA) at $4^{\circ} \mathrm{C}$ overnight. Next day, after washing membrane three times, then it was incubated with 1:1000 secondary antibody. Then the signals were detected using an enhanced chemiluminescence-detecting kit (Thermo Fisher, MA, USA), and the density of the bands was analyzed by using ImageJ.

2.8. Safety and Temperature Calculations. Finally, we conducted a safety test. We performed standard hematoxylineosin (HE, Sigma-Aldrich, St. Louis, Missouri, USA) staining of the brain and the CCI surgical area after the experiment in all test groups to determine that FUS would not cause further tissue damage and used mathematical modeling to determine the local temperature increase. Specifically, 12 FUS2-stimulated and Sham mice $(n=6 /$ group) were used for histological assessment. On the $21 \mathrm{st}$ day, 12 mice were cardiacally perfused with phosphatebuffered saline followed by $4 \%$ paraformaldehyde. The whole brains were then isolated and fixed in $4 \%$ paraformaldehyde (Sigma-Aldrich, St. Louis, Missouri, USA). The brain tissues were dehydrated, defatted, paraffinized, and serially sectioned at $4-\mu \mathrm{m}$ thicknesses using a pathologic microtome (Leica, RM2016, Wetzlar, Germany). We randomly selected the brain sections for observation under light microscopy. Upon the spread of ultrasonic energy into tissues, wave energy attenuation resulted from absorption or scattering 


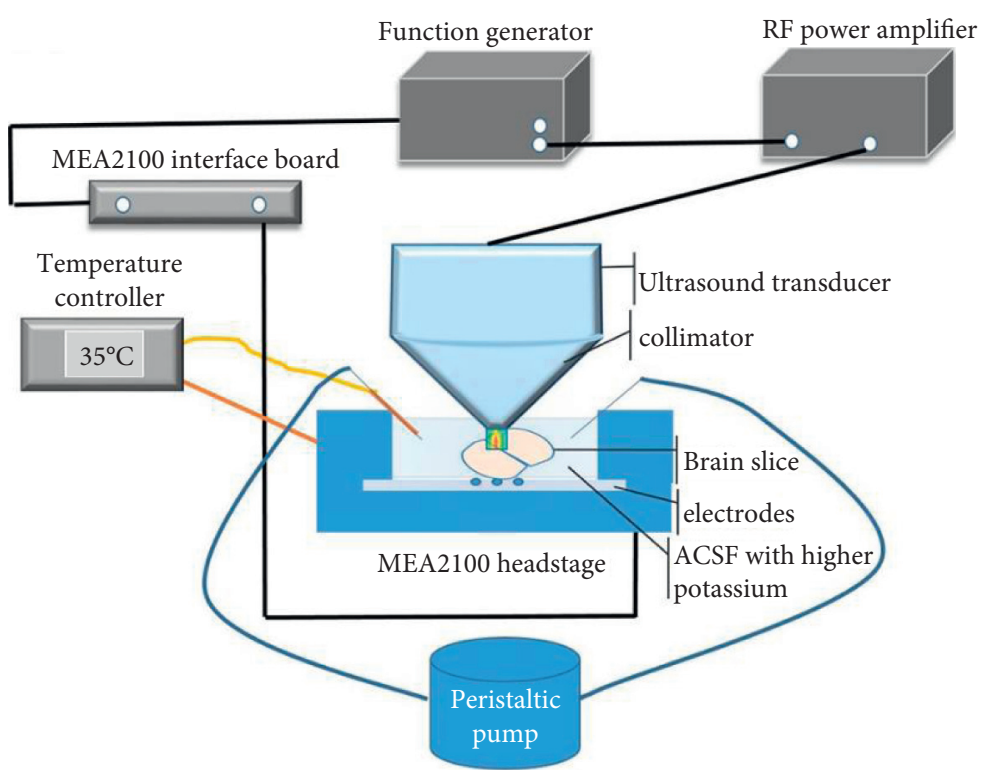

(a)

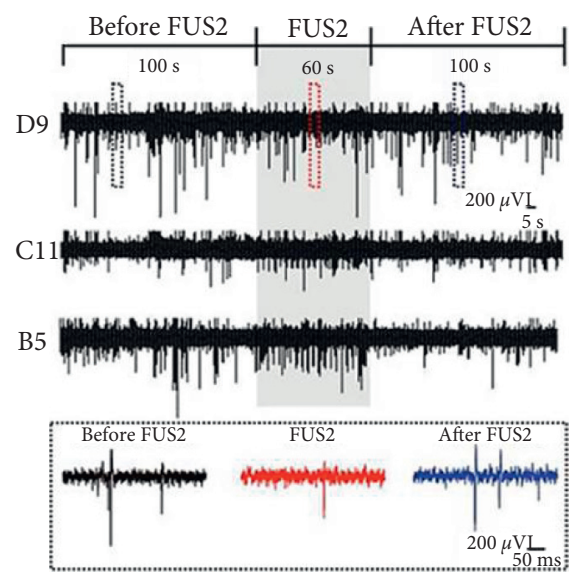

(b)

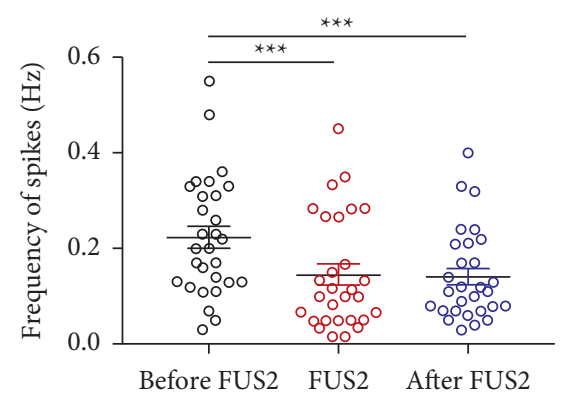

(c)

FIGURE 4: Ultrasound-induced inhibition of neuronal discharges from the ACC slices. Experimental schematics (a). Representative traces randomly selected of the neuronal discharges from the ACC slices obtained using MEA recording (b). Ultrasound stimulation substantially decreased the spike frequency of neuronal discharges (c) $\left({ }^{* * *} p<0.001\right.$ (prior to during: $p \leq 0.001$, prior to following: $\left.p=0.002\right)$ ).

with heat conversion or directional change, respectively. The balance between the absorbed energy and heat released results in an ultrasound-induced temperature increase that can be calculated using mathematical modeling techniques under different exposure conditions. Therefore, we calculated the ultrasound-induced local temperature increase to avoid local thermal damage using the following equation:

$$
Q=2 \alpha I_{\mathrm{TA}},
$$

where $\alpha$ is the absorption coefficient in the brain tissue, $I_{\mathrm{TA}}$ is the temporal-average intensity, and $Q$ is the heat generated per volume [30].

The FUS-induced maximum temperature increase $\left(\Delta T_{\max }\right)$ in the brain tissue could be described as follows without the heat loss:

$$
\Delta T_{\max }=\frac{\mathrm{Q} \Delta_{t}}{c_{v}}=\frac{\mathrm{Q} \Delta_{t}}{c_{\rho}},
$$

where $\Delta_{t}$ is the FUS exposure time and $C_{\nu}$ is the heat capacity per unit volume for brain tissue defined as the product of $\mathrm{C}$ (heat capacity in brain tissue: $3.6 \mathrm{~J} / \mathrm{g} /{ }^{\circ} \mathrm{C}$ ) and $\rho$ (density of brain tissue: $1028 \mathrm{~kg} / \mathrm{m}^{3}$ ) [31].
2.9. Statistical Analysis. We expressed all experimental data as mean \pm standard error (SME) and conducted analyses using independent sample $t$-tests and one-way ANOVA tests with Tukey's or Bonferroni's post hoc test for parametric analysis. All statistical analyses were performed in IBM $^{\circledR}$ SPSS ${ }^{\circledR}$ Statistics 23 (IBM Corp, Armonk, NY, USA), and the statistical significance was set at $p<0.05$.

\section{Results}

3.1. Transcranial FUS Stimulation Slowly Improved the Mechanical Withdraw Threshold in the Short-Term Experiment. The results showed an increase in the mechanical withdraw threshold of the operation side following the end of the FUS2 stimulation period (FUS1 group, $0.06 \pm 0.01 \mathrm{~g} ;$ FUS2 group, $0.32 \pm 0.09 \mathrm{~g}$; Sham group, $0.04 \pm 0.01 \mathrm{~g} ; p<0.01(p=0.003))$, indicating that the pain tolerance of mice on the surgical side had improved. FUS1 showed a similar but delayed and nonsignificant response. On the contralateral side, FUS2 exerted a similar effect; however, the outcome was not sustained (FUS1 group, $0.04 \pm 0.01 \mathrm{~g} ;$ FUS2 group, $0.15 \pm 0.05 \mathrm{~g}$; Sham group, $0.02 \pm 0.002 \mathrm{~g} ; \quad p<0.05 \quad(p=0.038) \quad($ Figure $2(\mathrm{~b}))$. 
Conversely, FUS1 stimulation induced a significant increase after 1 week (FUS1 group, $0.09 \pm 0.02 \mathrm{~g}$; FUS2 group, $0.08 \pm 0.02 \mathrm{~g} ; \quad$ Sham group, $0.02 \pm 0.005 \mathrm{~g} ; \quad p<0.05$ $(p=0.038)$ (Figure $2(c))$. There was substantial synchrony in the bilateral mechanical retraction domain following ultrasound stimulation and a significant improvement in the contralateral thermal withdrawal threshold following FUS2 stimulation (FUS1 group, $9.83 \pm 0.74 \mathrm{~s}$; FUS2 group, $12.67 \pm 1.12 \mathrm{~s}$; Sham group, $7.33 \pm 0.92 \mathrm{~s} ; p<0.01(p=0.003)$ (Figures 2(d) and 2(e)). Conversely, there were no significant changes in the sciatic nerve index and body weight (Figures $2(\mathrm{f})$ and $2(\mathrm{~g})$ ).

3.2. FUS Stimulation Promptly Improved the Mechanical Withdraw Threshold in the Long-Term Experiment. The results showed that the mechanical withdrawal threshold of the surgical side increased in the FUS group compared with that in the Sham group. This increase was sustained until the end of the FUS stimulation period (FUS group, $0.17 \pm 0.04 \mathrm{~g}$; Sham group, $0.06 \pm 0.02 \mathrm{~g} ; p<0.05(p=0.03))$ (Figure $3(\mathrm{~b}))$. There were no significant changes in the contralateral mechanical withdrawal threshold $(p=0.19)$ (Figure 3(b)), bilateral thermal withdrawal threshold (surgical side $p=0.21$, other side $p=0.25$ ) (Figures $3(\mathrm{~d}$ ) and $3(\mathrm{e})$ ), body weight $(p=0.93$ ) (Figure $3(\mathrm{~g})$ ), and sciatic nerve index $(p=0.49)$ (Figure $3(\mathrm{f}))$ between the FUS and the Sham groups.

\subsection{Ultrasound-Induced Inhibition of Neuronal Discharges} from the ACC Slices. Representative traces of spikes recorded during three phases in different channels randomly selected showed the ultrasound-induced changes using MEA recording (Figure 4(b)). The assessment of the 29 cells indicated that the spike frequencies in the ACC slices were effectively inhibited during ultrasound, which was sustained following ultrasound stimulation (prior to FUS2, $0.224 \pm 0.0023 \mathrm{~Hz}$; during FUS2, $0.145 \pm 0.0218 \mathrm{~Hz}$; following FUS2, $0.142 \pm 0.0176 \mathrm{~Hz},{ }^{* * *} p<0.001$ and prior to during: $p \leq 0.001$, prior to following: $p=0.002$ ), Student's paired $t$-test (Figure $4(\mathrm{c})$ ).

3.4. FUS-Induced Differences in Protein Expression Indicated Alterations in Pathways in the ACC. Protein profiling with a threshold of fold change of $>1.2$ and a $T$-test revealed differential up- and downregulations of 97 and 49 proteins, respectively; therefore, we observed a total of 146 protein expression changes. The subcellular structures of the proteins were mainly located in the cytoplasm, nucleus, and extracellular fluid (Figure 5(b)). Using EggNOG (EggNOG evolutionary genealogy of genes: Nonsupervised Orthologous Groups version 4.5.1, Computational Biology groupEMBL, Heidelberg), we found that the top 20 items (differential proteins) were involved in cellular processes, cellular signaling, and information storage and processing (Figure 5(a)). This suggests that ACC treatment with FUS alters cellular signal transduction and information processing involved in chronic pain. Further, enriched pathway analysis [32] (Cytoscape 3.7.2, Cytoscape Consortium) revealed that the differential proteins were mainly related to the endocrine, immune, and nervous systems (Figures 5(c) and $5(\mathrm{~d}))$.

3.5. FUS2-Induced Changes in the Expression of Hnrnph1, Hnrnpd, Snrpb, and Dhx16. Based on the plugin cytoHubba in Cytoscape, 4 key genes Hnrnph1, Hnrnpd, Snrpb, and Dhx16 were screened (Table S1). Western blotting results showed that HNRNPH1 and HNRNPD were significantly higher in the brain tissue of CCI mice compared with the normal group and the LUS stimulation group. Snrpb and Dhx16 expression shows no significant differences between CCI sham stimulation group and LUS stimulation group (Figure 6).

3.6. Safety and Temperature Evaluation. The results of $\mathrm{HE}$ staining of ACC brain tissue and local parts of CCI operation in the Control group, Sham group, and FUS2 group showed that the sciatic nerve had no neurofibrillation, swelling, or thickening. Further, there were no axon irregularities, the disappearance of changes, or myelin loosening, disintegration, or precise segmental loss. There was no substantial change in the number of nerve cells in the brain, especially the ACC. Moreover, there was no inflammatory cell infiltration between the stroma and blood vessels, nerve cell swelling, ulceration, pyknosis, coagulative necrosis, vesicular degeneration, or vacuolar degeneration (Figure 7).

\section{Discussion}

Transcranial FUS is a popular technique used for noninvasive neuromodulation in both animal models and humans [20]. Our findings indicated that ultrasound stimulation of the ACC increased the mechanical withdraw threshold in the CCI mice model in both the short- and long-term experiments. In the short experiment, the mechanical threshold of the intervention group started increasing at 2 weeks following the ultrasound, and we observed a substantial difference in the mechanical threshold between the intervention group and the Sham group at 3 weeks. In the long-term experiment, there was an early and sustained increase in the threshold following ultrasound stimulation. Similar to related studies in recent years, protein analysis results of the ACC region showed that it mainly involves the immune system and endocrine system considering the occurrence of pain, especially the occurrence of chronic pain, which may involve changes in the functions of the CNS [33-37]. In addition, HNRNPH1 and HNRNPD may become important regulatory targets for FUS to stimulate reduce neuropathic pain.

Ultrasound stimulation is a noninvasive technique that allows accurate targeting of different neurological diseases. Additionally, it is a safe and cheap therapeutic method [38-41]. The ultrasound is even safer in cases where pacemakers or other important implantable devices are not antimagnetic or antielectric. Compared with TMS and tDCS, focused ultrasound is easier to implement in the deep brain 

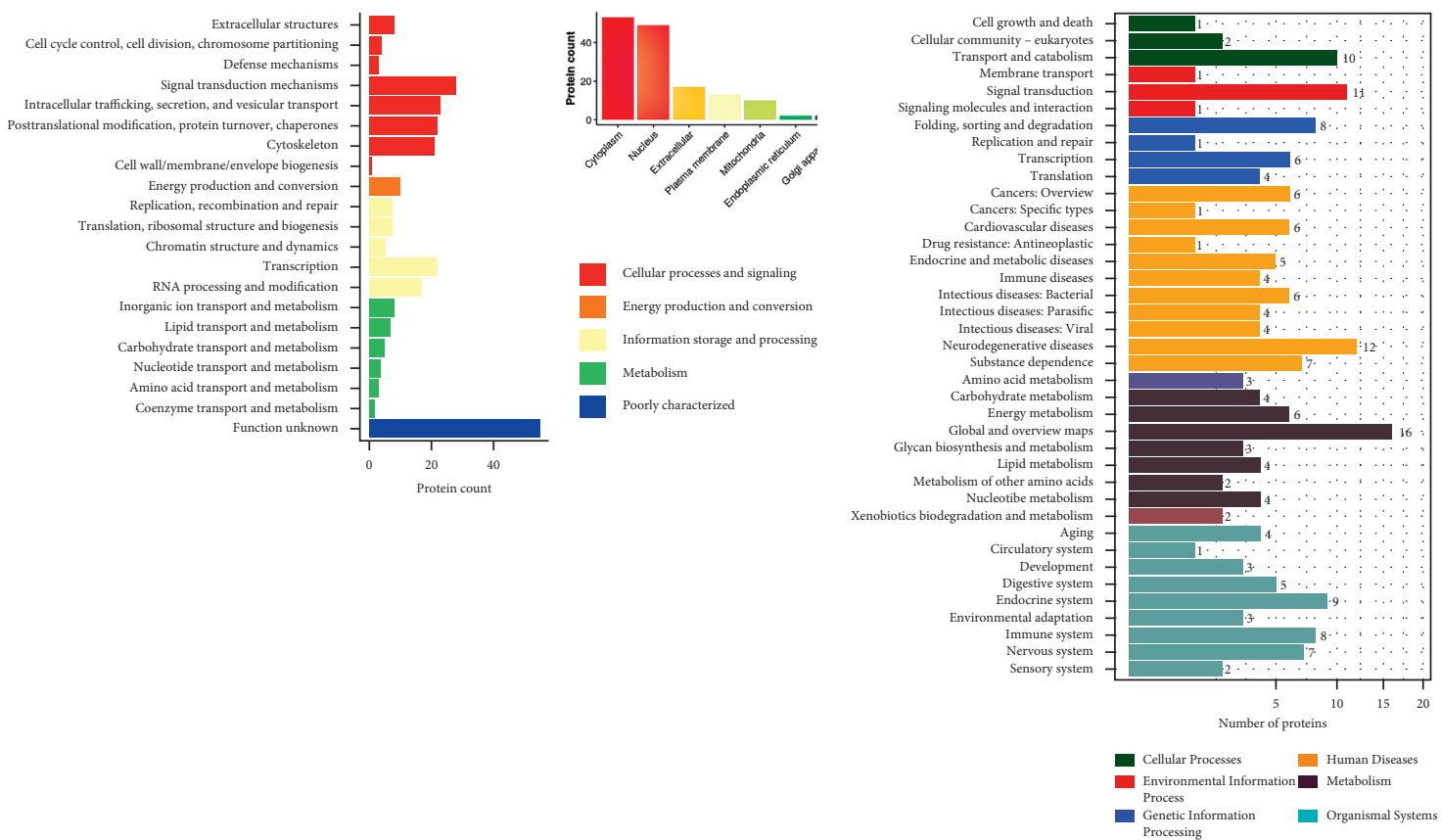

(a)

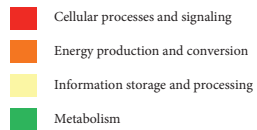

Metabolisn

Poorly characterized

(b)

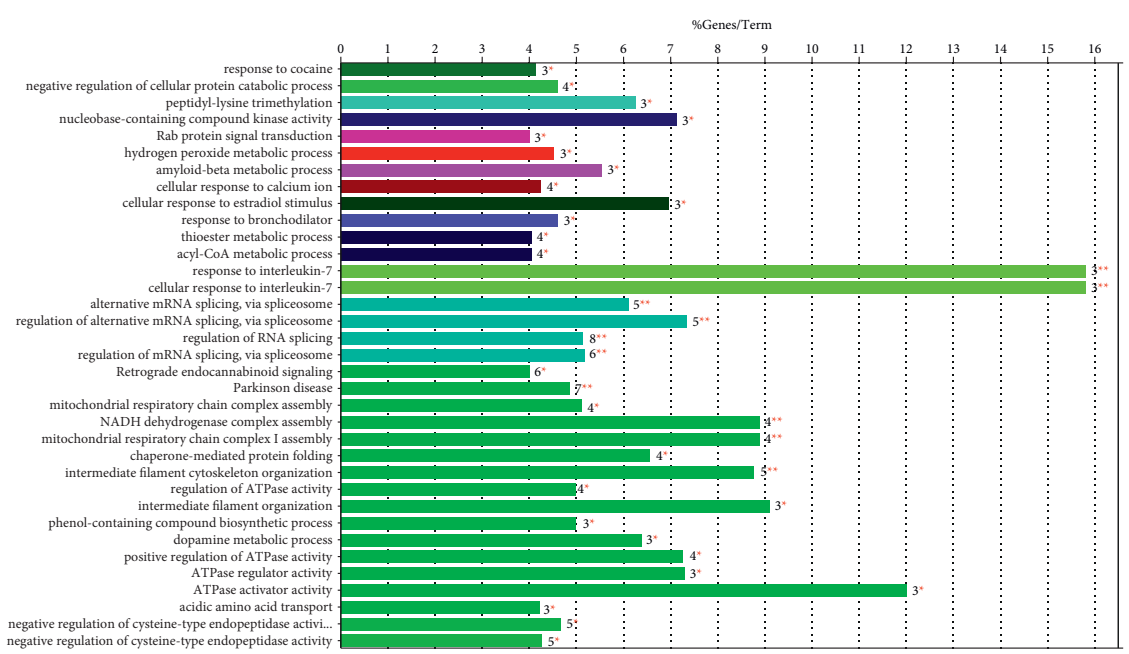

(c)

Figure 5: Continued. 


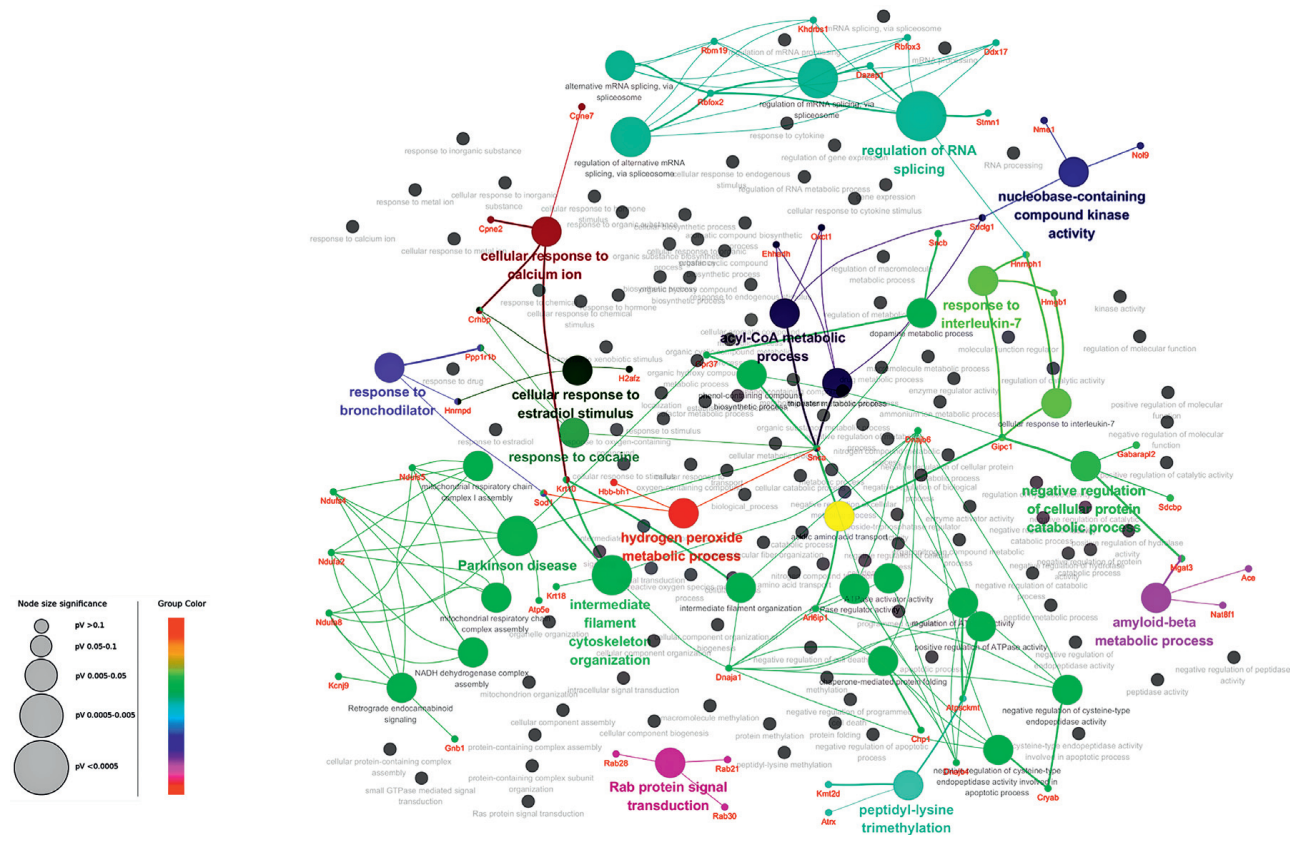

(d)

FIGURE 5: FUS-induced differences in protein expression indicated alterations in pathways in the ACC. EggNOG entry statistics (a). Subcellular location analysis (b). Visualized functional enrichment (c). Pathway analysis (d).
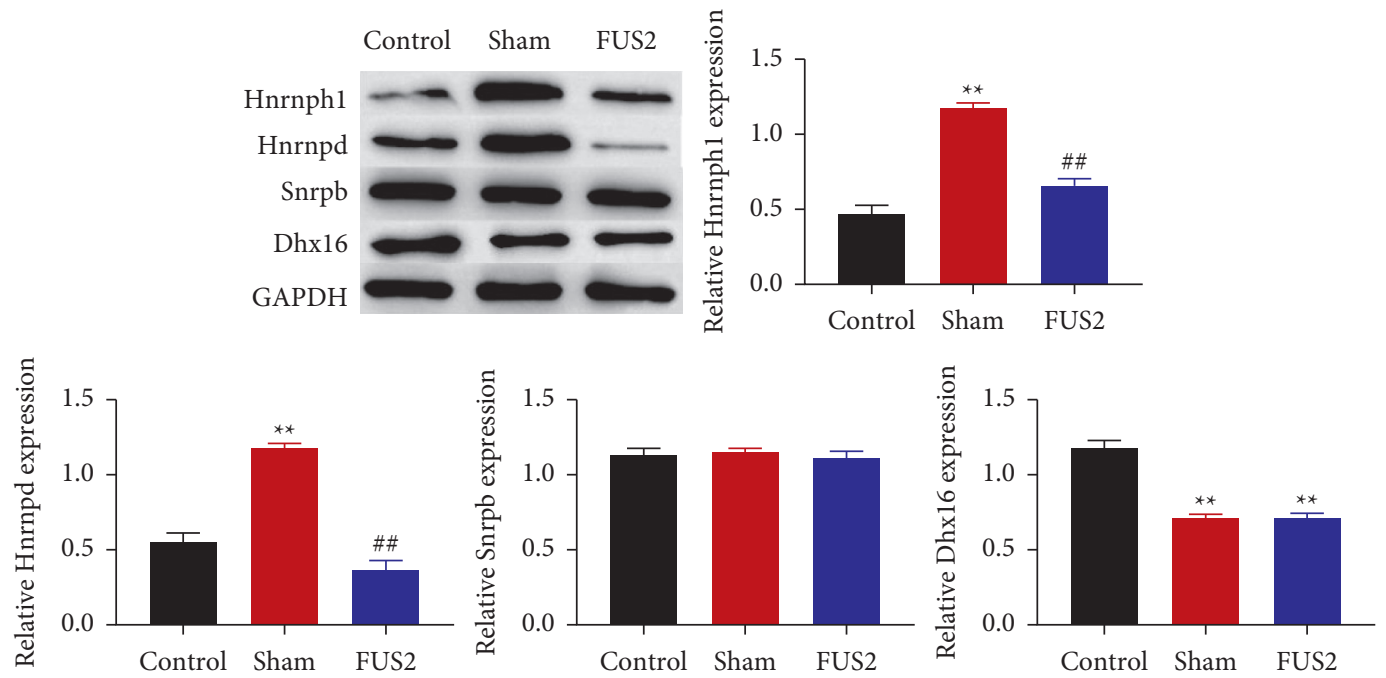

FIGURE 6: FUS2-induced changes in the expression of Hnrnph1, Hnrnpd, Snrpb, and Dhx16. The expression of Hnrnph1, Hnrnpd, Snrpb, and Dhx16 in tissue was measured by western blotting. * indicates statistical significance between the Sham group and the Control group, the FUS2 group and the Control group. " indicates statistical significance between the FUS2 group and the Sham group.

area, making it safer and more accurate and improving the effects of uneven skull bones in humans or large animals through combination with magnetic resonance imaging (MRI) [42]. Similar to magnetic stimulation, FUS can be used in regions of the peripheral and central nervous systems [43]. Moreover, they both require more effective treatment following repeated stimulation for pain management. The difference between the protocols is that the cortical M1 is a more effective target for central inhibition using repetitive transcranial magnetic stimulation $[16,44]$. At present, FUS is also widely used in larger animals. Low-intensity FUS modulated the excitability of regional brain tissues reversibly and safely in awake sheep [45]. In addition, FUS stimulation of female macaque monkeys under the guidance of MRI can double suppressive and excitative modulation of specific functional circuits [46]. FUS is different from other pharmacological or interventional therapies for acute and chronic pain. In our study, FUS stimulation of the ACC was effective in both the short- and long-term experiments, which suggests that FUS is a useful and noninvasive technique for pain management. Yang et al. reported increased neuronal excitability in ACC during nerve injury pain [47], 


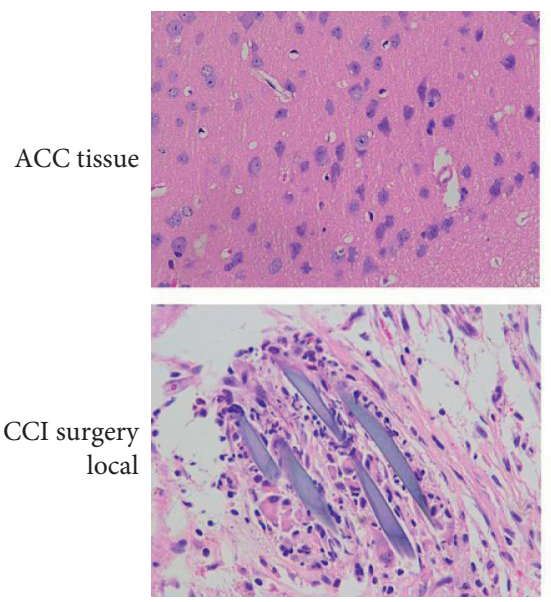

Control

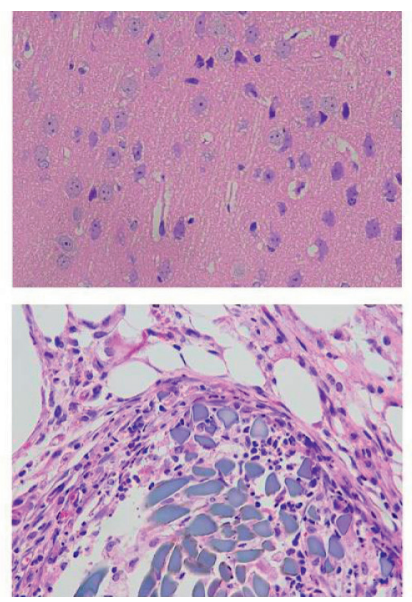

Sham
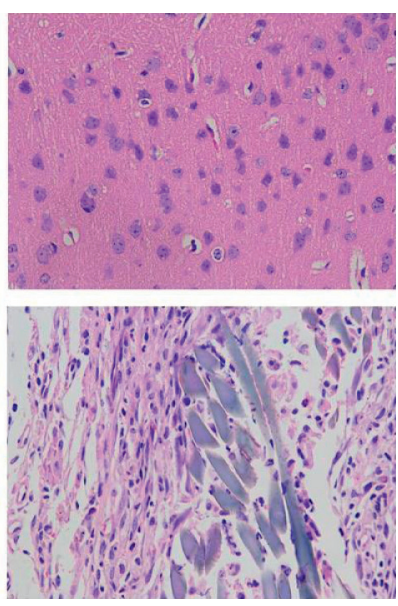

FUS2

FIgURE 7: Safety and temperature evaluation. HE staining of the ACC brain tissue and the CCI surgery local site $(\times 400)$.

especially in the bilateral ACC [48]. Similar to previous reports on other neuroregulatory methods (e.g., optogenetics) [13, 49-51], our MEA recordings revealed that FUS-induced reduction in the nerve excitability of the ACC in the brain slices was involved in pain attenuation, which also suggested that ACC could be an ideal target in CNS regulation of NP. Clennell et al. confirmed that there was a sustained effect until 8 hours after ultrasound stimulation [52], and we had found there was inhibition within 100s after ultrasound stimulation through MEA. In our experiment, the mechanical analgesic effect of ultrasound stimulation lasting for 3 weeks may be due to the continuous inhibition of nerve by repeated stimulation.

Peripheral nociceptor sensitization and durable synaptic plasticity in the CNS contribute to chronic pain in rodent models [53]. Although chronic and acute pain share common neural pathways [54, 55], establishing mechanisms for alleviating acute or chronic pain remains challenging. In a rodent NP model, central sensitization may be indicated by the characteristic potentiation of synaptic responses in the ACC and the development of allodynia at 1-2 weeks following nerve injury $[56,57]$. This may explain the earlier presentation of FUS effects in the long-term experiment. Contrastingly, in the short-term experiment, the FUS-induced effects required a longer time (two weeks following the surgery) to appear.

The difference in the time of onset of FUS effects between the short- and long-term experiments indicated that the ACC or the early stage of the pain was not the optimal target for pain improvement in the short-term experiments. Conversely, central sensitization may have been the main target for pain regulation in long-term tests. Our findings indicate that FUS stimulation of the ACC has a therapeutic effect on chronic pain following peripheral nerve injury through changes in the nervous and immune systems of the CNS. Similarly, a previous study that focused on similar peripheral damage reported that the emergence of central sensitization played a vital role in the resulting chronic pain [58]. The regulation of different brain regions would have different results from the literature [59-67], and ultrasound regulation of ACC improved mechanical retraction threshold in the CCI model in our study, suggesting that ultrasound stimulation of different target brain regions can be considered to obtain better curative effect according to the different pain symptoms.

The occurrence and development of NP are caused by many factors and the disorder of genes regulation. Recent studies have found that FUS stimulation can improve the progress of NP by regulating the disordered genes [68]. In this study, we found four key genes Hnrnph1, Hnrnpd, Snrpb, and Dhx16 by analyzing the protein profile of longterm experimental mice. Further research found that Hnrnph1 and Hnrnpd are highly expressed in the brain tissue of NP mice, but downregulated in normal mouse brain tissue and FUS stimulation. HNRNPH1 and HNRNPD (heterogeneous nuclear ribonucleoprotein $\mathrm{D}$ ) is a multifunctional RNA binding protein (RBP) with roles in regulation of alternative splicing, mRNA transcription, RNA stability, RNA localization, and regulation of target transcript translation. HNRNPH1 is abnormally overexpressed in a variety of tumors. Previous studies have confirmed that the high expression of HNRNPH1 can promote tumor development by inhibiting tumor suppressor genes [69]. However, the role of HNRNPH1 in NP has not been investigated. HNRNPD participates in the regulation of cell oxidative stress and inflammation. And it is also related to the cross-regulation of inflammation [70]. In addition, HNRNPD participates in the apoptosis of diabetic cells [71]. But the role of HNRNPD in NP has not been reported. According to the results of this experiment, we speculate that HNRNPH1 and HNRNPD may play a key role in NP, and, at the same time, FUS stimulation can directly or indirectly downregulate the levels of HNRNPH1 and HNRNPD to improve the development of NP. However, to determine the regulatory mechanism of HNRNPH1 and HNRNPD in NP, further research and investigation are needed.

Ultrasound waves have mechanical, cavitation, and thermal effects on biological tissue. The thermal effects of high-intensity FUS (HIFU $>200 \mathrm{~W} / \mathrm{cm}^{2}$ ) are reported to cause coagulative necrosis of brain tissue through the intact 
skull $[72,73]$. Findings on HE staining and local temperature increase $\left(\Delta T<0.1^{\circ} \mathrm{C}\right)$ showed that the treatment was safe and did not cause tissue damage to the target tissue and the surrounding. Moreover, the maximum negative peak pressure was much lower than the inertial cavitation threshold (40 MPa), which prevented tissue damage [74].

In conclusion, previous studies have indicated the safety of transcranial ultrasound stimulation, which is consistent with the results of other studies $[21,75,76]$. Our findings indicate that FUS effectively alleviates mechanical NP via ACC inhibition, especially in the chronic state. The underlying mechanisms may be associated with several central sensitization stages, suggesting that different protocols may be more appropriate for different NP stages. Protein analysis in the long-term experiment demonstrated that FUS-induced neuromodulation of the ACC altered the immune function and several pathways involved in central sensitization. These results suggested great potential for clinical translation. Particularly, it is of significance to select different intervention programs at different clinical stages. However, ultrasound is prone to be off-target due to the heterogeneity of the skull, and MRI is needed to correct the ultrasound stimulation in humans or large animals. Based on ethical principles, we could not use a larger sample of mice to verify every parameter, and there may be more reasonable parameters inducing different effects. Future studies should investigate targeted brain regions, appropriate time points, and response pathways to identify the mechanisms underlying FUS treatment of NP.

\section{Data Availability}

The data used to support the findings of this study are available from the corresponding author upon reasonable request.

\section{Disclosure}

The funding sources had no role in the study design; in the collection, analysis, and interpretation of data; in the writing of the report; or in the decision to submit the article for publication.

\section{Conflicts of Interest}

The authors declare that there are no conflicts of interest regarding the publication of this study.

\section{Acknowledgments}

The authors would like to thank Dr. Mian Chen (Biomedical Engineering Department of Shenzhen University) for providing his laser transmitter and technical help. This work was supported by the National Natural Science Foundation of China (grant nos. 81960421, 81660381, 81527901, 11774371, 11574341, 11674347, and 12004410), Guangdong-Hong Kong-Macao Greater Bay Area Center for Brain Science and Brain-Inspired Intelligence Fund (no. 2019024), Guangdong grant "Key Technologies for Treatment of Brain Disorders" (no. 2018B030332001), CAS Key Laboratory of Health
Informatics Fund (2011DP173015), and Doctoral Innovation Foundation of Kunming Medical University (no. 2019D007).

\section{Supplementary Materials}

Table S1: cytoHubba function results. (Supplementary Materials)

\section{References}

[1] S.-F. Cho, K.-M. Rau, Y.-Y. Shao et al., "Patients with head and neck cancer may need more intensive pain management to maintain daily functioning: a multi-center study," Supportive Care in Cancer, vol. 27, no. 5, pp. 1663-1672, 2019.

[2] J. Gierthmühlen and R. Baron, "Neuropathic pain," Seminars in Neurology, vol. 36, pp. 462-468, 2016.

[3] Ó. A. Steingrímsdóttir, T. Landmark, G. J. Macfarlane, and C. S. Nielsen, "Defining chronic pain in epidemiological studies: a systematic review and meta-analysis," Pain, vol. 158, no. 11, pp. 2092-2107, 2017.

[4] L. E. Simons, I. Elman, and D. Borsook, "Psychological processing in chronic pain: a neural systems approach," Neuroscience \& Biobehavioral Reviews, vol. 39, pp. 61-78, 2014.

[5] D. Moulin, A. Boulanger, A. CLArK et al., "Pharmacological management of chronic neuropathic pain: revised consensus statement from the Canadian Pain Society," Pain Research and Management, vol. 19, no. 6, pp. 328-335, 2014.

[6] X. Moisset, D. Bouhassira, J. Avez Couturier et al., "Pharmacological and non-pharmacological treatments for neuropathic pain: systematic review and French recommendations," Revue Neurologique, vol. 176, no. 5, pp. 325-352, 2020.

[7] C.-H. Hung, P.-C. Huang, J.-I. Tzeng, J.-J. Wang, and Y.-W. Chen, "Therapeutic ultrasound and treadmill training suppress peripheral nerve injury-induced pain in rats," Physical Therapy, vol. 96, no. 10, pp. 1545-1553, 2016.

[8] W. Jiang, Y. Wang, J. Tang et al., "Low-intensity pulsed ultrasound treatment improved the rate of autograft peripheral nerve regeneration in rat," Scientific Reports, vol. 6, no. 1, Article ID 22773, 2016.

[9] S. Hameroff, M. Trakas, C. Duffield et al., "Transcranial ultrasound (TUS) effects on mental states: a pilot study," Brain Stimulation, vol. 6, no. 3, pp. 409-415, 2013.

[10] J. Spooner, H. Yu, C. Kao, K. Sillay, and P. Konrad, "Neuromodulation of the cingulum for neuropathic pain after spinal cord injury. Case report," Journal of Neurosurgery, vol. 107, no. 1, pp. 169-172, 2007.

[11] S. M. Farrell, A. Green, and T. Aziz, "The current state of deep brain stimulation for chronic pain and its context in other forms of neuromodulation," Brain Sciences, vol. 8, 2018.

[12] T. Chen, K. Koga, G. Descalzi et al., "Postsynaptic potentiation of corticospinal projecting neurons in the anterior cingulate cortex after nerve injury," Molecular Pain, vol. 10, p. 33, 2014.

[13] L. Gu, M. L. Uhelski, S. Anand et al., "Pain inhibition by optogenetic activation of specific anterior cingulate cortical neurons," PLoS One, vol. 10, Article ID e0117746, 2015.

[14] W. Masocha, "Astrocyte activation in the anterior cingulate cortex and altered glutamatergic gene expression during paclitaxel-induced neuropathic pain in mice," PeerJ, vol. 3, Article ID e1350, 2015.

[15] H. C. Moon, W. I. Heo, Y. J. Kim et al., "Optical inactivation of the anterior cingulate cortex modulate descending pain 
pathway in a rat model of trigeminal neuropathic pain created via chronic constriction injury of the infraorbital nerve," Journal of Pain Research, vol. 10, pp. 2355-2364, 2017.

[16] H. Kumru, S. Albu, J. Vidal, and J. M. Tormos, "Effectiveness of repetitive trancranial or peripheral magnetic stimulation in neuropathic pain," Disability \& Rehabilitation, vol. 39, no. 9, pp. 856-866, 2017.

[17] V. Levi, R. Cordella, A. D'Ammando et al., "Dorsal anterior cingulate cortex (ACC) deep brain stimulation (DBS): a promising surgical option for the treatment of refractory thalamic pain syndrome (TPS)," Acta Neurochirurgica, vol. 161, no. 8, pp. 1579-1588, 2019.

[18] O. Naor, S. Krupa, and S. Shoham, "Ultrasonic neuromodulation," Journal of Neural Engineering, vol. 13, Article ID 031003, 2016.

[19] R. F. Dallapiazza, K. F. Timbie, S. HolmberG et al., "Noninvasive neuromodulation and thalamic mapping with lowintensity focused ultrasound," Journal of Neurosurgery, vol. 128, no. 3, pp. 875-884, 2018.

[20] L. Di Biase, E. Falato, and V. Di Lazzaro, "Transcranial focused ultrasound (tFUS) and transcranial unfocused ultrasound (tUS) neuromodulation: from theoretical principles to stimulation practices," Frontiers in Neurology, vol. 10, p. 549, 2019.

[21] X. Huang, H. Zheng, Z. Lin et al., “Transcranial low-intensity pulsed ultrasound modulates structural and functional synaptic plasticity in rat Hippocampus," IEEE Transactions on Ultrasonics, Ferroelectrics, and Frequency Control, vol. 66, no. 5, pp. 930-938, 2019.

[22] H. Zhou, L. Niu, L. Meng et al., "Noninvasive ultrasound deep brain stimulation for the treatment of Parkinson's disease model mouse," Research: Ideas for Today's Investors, vol. 2019, Article ID 1748489, 13 pages, 2019.

[23] G. J. Bennett and Y.-K. Xie, "A peripheral mononeuropathy in rat that produces disorders of pain sensation like those seen in man," Pain, vol. 33, no. 1, pp. 87-107, 1988.

[24] V. V. Monte-Raso, C. H. Barbieri, N. Mazzer, A. C. Yamasita, and G. Barbieri, "Is the sciatic functional index always reliable and reproducible?" Journal of Neuroscience Methods, vol. 170, no. 2, pp. 255-261, 2008.

[25] A. Becchetti, F. Gullo, G. Bruno, E. Dossi, M. Lecchi, and E. Wanke, "Exact distinction of excitatory and inhibitory neurons in neural networks: a study with GFP-GAD67 neurons optically and electrophysiologically recognized on multielectrode arrays," Frontiers in Neural Circuits, vol. 6, p. 63, 2012.

[26] Q. Jiang, G. Li, H. Zhao et al., "Temporal neuromodulation of retinal ganglion cells by low-frequency focused ultrasound stimulation," IEEE Transactions on Neural Systems and Rehabilitation Engineering, vol. 26, no. 5, pp. 969-976, 2018.

[27] S. Wiese, K. A. Reidegeld, H. E. Meyer, and B. Warscheid, "Protein labeling by iTRAQ: a new tool for quantitative mass spectrometry in proteome research," Proteomics, vol. 7, no. 3, pp. 340-350, 2007.

[28] G. Paxinos and K. B. J. Franklin, "The mouse brain in stereotaxic coordinates," Elsevier, Amsterdam, Netherlands, 2nd edition, 2001.

[29] J. R. Wisniewski, A. Zougman, N. Nagaraj, and M. Mann, "Universal sample preparation method for proteome analysis," Nature Methods, vol. 6, pp. 359-362, 2009.

[30] D. Zhang, H. Li, J. Sun et al., "Antidepressant-like effect of low-intensity transcranial ultrasound stimulation," IEEE Transactions on Biomedical Engineering, vol. 66, no. 2, pp. 411-420, 2019.
[31] W. D. O’brien JR., "Ultrasound-biophysics mechanisms," Progress in Biophysics and Molecular Biology, vol. 93, pp. 212-255, 2007.

[32] P. Shannon, A. Markiel, O. Ozier et al., "Cytoscape: a software environment for integrated models of biomolecular interaction networks," Genome Research, vol. 13, no. 11, pp. 2498-2504, 2003.

[33] F. Marchand, M. Perretti, and S. B. Mcmahon, "Role of the immune system in chronic pain," Nature Reviews Neuroscience, vol. 6, no. 7, pp. 521-532, 2005.

[34] R. Geenen and J. W. J. Bijlsma, "Deviations in the endocrine system and brain of patients with fibromyalgia: cause or consequence of pain and associated features?" Annals of the New York Academy of Sciences, vol. 1193, no. 1, pp. 98-110, 2010.

[35] K. Ren and R. Dubner, "Interactions between the immune and nervous systems in pain," Nature Medicine, vol. 16, no. 11, pp. 1267-1276, 2010.

[36] F. Tennant, "The physiologic effects of pain on the endocrine system," Pain and Therapy, vol. 2, no. 2, pp. 75-86, 2013.

[37] S. K. Totsch and R. E. Sorge, "Immune system involvement in specific pain conditions," Molecular Pain, vol. 13, Article ID 1744806917724559, 2017.

[38] M. E. Downs, S. A. Lee, G. Yang, S Kim, Q. Wang, and E. E. Konofagou, "Non-invasive peripheral nerve stimulation via focused ultrasound in vivo," Physics in Medicine and Biology, vol. 63, p. 035011, 2018.

[39] K. Eguchi, T. Shindo, K. Ito et al., "Whole-brain low-intensity pulsed ultrasound therapy markedly improves cognitive dysfunctions in mouse models of dementia - crucial roles of endothelial nitric oxide synthase," Brain Stimulation, vol. 11, no. 5, pp. 959-973, 2018.

[40] V. Cotero, Y. Fan, T. Tsaava et al., "Noninvasive sub-organ ultrasound stimulation for targeted neuromodulation," $\mathrm{Na}$ ture Communications, vol. 10, no. 1, p. 952, 2019.

[41] H. Zhou, L. Niu, X. Xia et al., "Wearable ultrasound improves motor function in an MPTP mouse model of Parkinson's disease," IEEE Transactions on Biomedical Engineering, vol. 66, 2019.

[42] A. Bystritsky, A. S. Korb, P. K. Douglas et al., "A review of lowintensity focused ultrasound pulsation," Brain Stimulation, vol. 4, no. 3, pp. 125-136, 2011.

[43] E. Hattapoglu, I. Batmaz, B. Dilek, M. Karakoç, S. Em, and R. Çevik, "Efficiency of pulsed electromagnetic fields on pain, disability, anxiety, depression, and quality of life in patients with cervical disc herniation: a randomized controlled study," Turkish Journal of Medical Sciences, vol. 49, pp. 1095-1101, 2019.

[44] A. Leung, M. Donohue, R. Xu et al., "rTMS for suppressing neuropathic pain: a meta-analysis," The Journal of Pain, vol. 10, no. 12, pp. 1205-1216, 2009.

[45] H. C. Kim, W. Lee, J. Kunes et al., "Transcranial focused ultrasound modulates cortical and thalamic motor activity in awake sheep," Scientific Reports, vol. 11, Article ID 19274, 2021.

[46] P.-F. Yang, M. A. Phipps, S. Jonathan et al., "Bidirectional and state-dependent modulation of brain activity by transcranial focused ultrasound in non-human primates," Brain Stimulation, vol. 14, no. 2, pp. 261-272, 2021.

[47] Z. Yang, Q. Tan, D. Cheng et al., "The changes of intrinsic excitability of pyramidal neurons in anterior cingulate cortex in neuropathic pain," Frontiers in Cellular Neuroscience, vol. 12, p. 436, 2018. 
[48] R. Zhao, H. Zhou, L. Huang et al., "Neuropathic pain causes pyramidal neuronal hyperactivity in the anterior cingulate cortex," Frontiers in Cellular Neuroscience, vol. 12, p. 107, 2018.

[49] M. F. Dossantos, N. Ferreira, R. L. Toback, A. C. Carvalho, and A. F. DaSilva, "Potential mechanisms supporting the value of motor cortex stimulation to treat chronic pain syndromes," Frontiers in Neuroscience, vol. 10, p. 18, 2016.

[50] D. L. Juarez-Salinas, J. M. Braz, A. Etlin, S. Gee, V. Sohal, and A. I. Basbaum, "GABAergic cell transplants in the anterior cingulate cortex reduce neuropathic pain aversiveness," Brain, vol. 142, no. 9, pp. 2655-2669, 2019.

[51] D. J. Lee, C. S. Lozano, R. F. Dallapiazza, and A. M. Lozano, "Current and future directions of deep brain stimulation for neurological and psychiatric disorders," Journal of Neurosurgery, vol. 131, no. 2, pp. 333-342, 2019.

[52] B. Clennell, T. G. J. Steward, M. Elley et al., "Transient ultrasound stimulation has lasting effects on neuronal excitability," Brain Stimulation, vol. 14, no. 2, pp. 217-225, 2021.

[53] M. Zhuo, "Cortical excitation and chronic pain," Trends in Neurosciences, vol. 31, no. 4, pp. 199-207, 2008.

[54] A. V. Apkarian, M. C. Bushnell, R.-D. Treede, and J.-K. Zubieta, "Human brain mechanisms of pain perception and regulation in health and disease," European Journal of Pain, vol. 9, no. 4, p. 463, 2005.

[55] M. C. Bushnell, M. Čeko, and L. A. Low, "Cognitive and emotional control of pain and its disruption in chronic pain," Nature Reviews Neuroscience, vol. 14, no. 7, pp. 502-511, 2013.

[56] X.-Y. Li, H.-G. Ko, T. Chen et al., "Alleviating neuropathic pain hypersensitivity by inhibiting PKMzeta in the anterior cingulate cortex," Science, vol. 330, no. 6009, pp. 1400-1404, 2010.

[57] K. Koga, G. Descalzi, T. Chen et al., "Coexistence of two forms of LTP in ACC provides a synaptic mechanism for the interactions between anxiety and chronic pain," Neuron, vol. 85, no. 2, pp. 377-389, 2015.

[58] C. Den Boer, L. Dries, B. Terluin et al., "Central sensitization in chronic pain and medically unexplained symptom research: a systematic review of definitions, operationalizations and measurement instruments," Journal of Psychosomatic Research, vol. 117, pp. 32-40, 2019.

[59] H. Sun and V. Neugebauer, "mGluR1, but not mGluR5, activates feed-forward inhibition in the medial prefrontal cortex to impair decision making," Journal of Neurophysiology, vol. 106, no. 2, pp. 960-973, 2011.

[60] L. Luongo, V. De Novellis, L. Gatta et al., "Role of metabotropic glutamate receptor 1 in the basolateral amygdaladriven prefrontal cortical deactivation in inflammatory pain in the rat," Neuropharmacology, vol. 66, pp. 317-329, 2013.

[61] C. Giordano, L. Cristino, L. Luongo et al., "TRPV1-dependent and -independent alterations in the limbic cortex of neuropathic mice: impact on glial caspases and pain perception," Cerebral Cortex, vol. 22, no. 11, pp. 2495-2518, 2012.

[62] W. E. Wang, R. L. M. Ho, B. Gatto et al., "Cortical dynamics of movement-evoked pain in chronic low back pain," The Journal of Physiology, vol. 599, no. 1, pp. 289-305, 2021.

[63] M. Zhuo, "Neural mechanisms underlying anxiety-chronic pain interactions," Trends in Neurosciences, vol. 39, no. 3, pp. 136-145, 2016.

[64] K. Carlsson, J. Andersson, P. Petrovic, K. M. Petersson, A. Öhman, and M. Ingvar, "Predictability modulates the affective and sensory-discriminative neural processing of pain," NeuroImage, vol. 32, no. 4, pp. 1804-1814, 2006.
[65] C. A. Brown, B. Seymour, Y. Boyle, W. El-Deredy, and A. K. P. Jones, "Modulation of pain ratings by expectation and uncertainty: behavioral characteristics and anticipatory neural correlates," Pain, vol. 135, no. 3, pp. 240-250, 2008.

[66] G.-Q. Wang, C. Cen, C. Li et al., "Deactivation of excitatory neurons in the prelimbic cortex via Cdk5 promotes pain sensation and anxiety," Nature Communications, vol. 6, no. 1, p. 7660, 2015.

[67] B. W. Badran, K. A. Caulfield, S. Stomberg-Firestein et al., "Sonication of the anterior thalamus with MRI-Guided transcranial focused ultrasound (tFUS) alters pain thresholds in healthy adults: a double-blind, sham-controlled study," Brain Stimulation, vol. 13, no. 6, pp. 1805-1812, 2020.

[68] Y. H. Liao, B. Wang, M. X. Chen, Y. Liu, and Li-J. Ao, "LIFU alleviates neuropathic pain by improving the $\mathrm{KCC}(2)$ expression and inhibiting the CaMKIV-KCC(2) pathway in the L4-L5 section of the spinal cord," Neural Plasticity and Neuropathic Pain, vol. 2021, Article ID 6659668, 2021.

[69] M. Liu, L. Yang, X. Liu et al., "HNRNPH1 is a novel regulator of cellular proliferation and disease progression in chronic myeloid leukemia," Frontiers in Oncology, vol. 11, Article ID 682859, 2021.

[70] T. Ishii, H. Hayakawa, T. Sekiguchi, N. Adachi, and M. Sekiguchi, "Role of Auf1 in elimination of oxidatively damaged messenger RNA in human cells," Free Radical Biology and Medicine, vol. 79, pp. 109-116, 2015.

[71] E. C. Vanzela and A. K. Cardozo, "Is ARE/poly(U)-binding factor 1 (AUF1) a new player in cytokine-mediated beta cell apoptosis?” Diabetologia, vol. 55, no. 6, pp. 1572-1576, 2012.

[72] W. J. Elias, D. Huss, T. Voss et al., "A pilot study of focused ultrasound thalamotomy for essential tremor," New England Journal of Medicine, vol. 369, no. 7, pp. 640-648, 2013.

[73] V. Krishna, F. Sammartino, P. Agrawal et al., "Prospective tractography-based targeting for improved safety of focused ultrasound thalamotomy," Neurosurgery, vol. 84, pp. 160-168, 2019.

[74] D. Dalecki, "Mechanical bioeffects of ultrasound," Annual Review of Biomedical Engineering, vol. 6, no. 1, pp. 229-248, 2004.

[75] T. Deffieux, Y. Younan, N. Wattiez, M. Tanter, P. Pouget, and J.-F. Aubry, "Low-intensity focused ultrasound modulates monkey visuomotor behavior," Current Biology, vol. 23, no. 23, pp. 2430-2433, 2013.

[76] Z. Zhang, W. Qiu, H. Gong et al., "Low-intensity ultrasound suppresses low-Mg(2+)-induced epileptiform discharges in juvenile mouse hippocampal slices," Journal of Neural Engineering, vol. 16, Article ID 036006, 2019. 